\title{
Pneumonia:
}

\section{A focus on treatment}

Tn 1680, John Bunion referred to tuberculosis as the Captain of the Men of Death; Sir William Osler later borrowed this phrase to describe pneumonia. Of the various infections that physicians may encounter, pneumonia is the one infection that virtually all must deal with on a regular basis regardless of their pattern of practice.

Pneumonia is a common condition, but has serious consequences in terms of morbidity and mortality. The sequelae associated with pneumonia have an impact on the individual, society and the economy; estimates based on data from the United States suggest that in that country the yearly cost of treating this illness is $\$ 23$ billion.

Pneumonia is usually considered as being either community acquired (CAP) (including nursing home) or hospital acquired (HAP) pneumonia. The latter is generally defined as infection occurring $48 \mathrm{~h}$ or more after admission to hospital and not incubating at the time of admission.

In this issue, two conference reports that deal with initial antimicrobial treatment of CAP and HAP are reproduced $(1,2)$. The reader will notice that both employ regimens that are reasonably broad in terms of their spectrum of antimicrobial activity. The reasons for this become clear when we consider the pathogenesis of pneumonia, the changes in its epidemiology and the problems one faces when trying to diagnose it.

The etiological agents responsible for CAP differ from those in HAP. In a recent review, findings of three studies showed that in $43 \%$ of cases of CAP, the etiology was unknown, in $8 \%$ it was polymicrobial and in the remaining $49 \%$ a wide variety of pathogens ranging from Streptococcus pneumoniae to Mycoplasma pneumoniae and Chlamydia pneumoniae were responsible (3). In HAP, the predominant pathogens are Gram-negative rods and certain Gram-positive cocci. The former include Enterobacter species, Escherichia coli, Haemophilus influenzae, Klebsiella and Proteus species and Serratia marcescens, while the latter include Staphylococcus aureus and S pneumoniae. In more severe cases, Pseudomonas and Acinetobacter species must also be included.

The predominance of Gram-negative bacilli as causative pathogens in nosocomial cases relates to changes in the nature of the bacteria that colonize the oropharynx of individuals with underlying disease and to the mechanisms by which pathogens reach the lung. Bacteria may gain access to the lower respiratory tract by a variety of means, including inhalation, hematogenous spread, translocation from the gastrointestinal tract and aspiration. Of these, aspiration is the most common.

Gram-negative bacilli colonize the oropharynx of fewer than $10 \%$ of healthy people; however, this figure jumps to $75 \%$ in those who are critically ill (4). The net result is that ill individuals aspirate much more virulent organisms during sleep than do healthy individuals, with pneumonia being one of the possible outcomes.

Changes in the epidemiology of pneumonia are evident in CAP and nosocomial cases. In the former, there appears to be a higher incidence of infection with so-called atypical pathogens such as $M$ pneumoniae and $C$ pneumoniae, while in nosocomial cases, infection with multiply resistant Gram-negative bacilli and methicillin-resistant $S$ aureus are becoming increasingly important.

The diagnosis of pneumonia is certainly far from straightforward. In CAP cases, it was often taught that certain symptoms, such as a cough productive of purulent sputum and pleuritic chest pain, were suggestive of 'typical' pneumonia as might be caused by classic bacterial pathogens such as $S$ pneumoniae. On the other hand, symptoms such as a nonproductive cough or a cough productive of mucoid sputum were suggestive of 'atypical' pneumonia and infection with organisms such as M pneumoniae or $C$ pneumoniae. A study by Fang et al (5), however, showed that the etiological agent could not be determined based on the clinical symptoms or physical signs.

In HAP cases, the physician is not only faced with a wide variety of possible pathogens to consider but also must often differentiate between infective and noninfective causes of fever and pulmonary infiltrates such as pulmonary emboli, adult respiratory distress syndrome and pulmonary drug reactions.

Given these considerations, it is clear that developing guidelines for initial treatment regimens of pneumonia is not a straightforward task. A variety of approaches was carefully considered, but ultimately it was felt that, to be useful, the approach chosen must be based on variables that are likely to predict specific pathogens and the patient's clinical course.

The variables used as the basis for initial treatment of CAP and HAP are, first for CAP:

- the severity of illness upon clinical presentation;

- whether the pneumonia was acquired in the community setting or in a nursing home;

- the presence or absence of comorbid illness (or age older than 65 years); and 
- whether treatment is to be given in the community setting, the nursing home or following admission of the patient to a hospital.

and for HAP:

- mild to moderate pneumonia with no unusual risk factors;

- mild to moderate pneumonia with risk factors; and

- severe pneumonia.

The guidelines offer a number of possible regimens for the various clinical scenarios. One could argue that fewer choices should have been offered or that more specific regimens should have been selected. It is apparent, however, that there is some regional or geographic variation in terms of epidemiological patterns as well as susceptibilities of microorganisms to various antimicrobials. By providing a number of alternatives, the individual physician can then choose the best possible regimen, keeping in mind the local factors that may influence the choice of drugs. It is stressed that these are meant as guidelines only for initial antimicrobial management and should be modified when necessary if appropriate culture and sensitivity results become available.

Since pneumonia is a difficult disease to diagnose and treat, physicians have found its management frustrating, to say the least. Because of their pragmatic and practical approach, these guidelines have been extremely well received by family physicians and specialists alike. Using the Canadian guidelines as a foundation, the American Thoracic Society recently published its own guidelines for CAP and is currently developing nosocomial pneumonia guidelines, which are also based on the Canadian document $(1,2,6)$.

\section{REFERENCES}

1. Mandell LA, Niederman M, The Canadian Community Acquired Pneumonia Consensus Conference Group. Antimicrobial treatment of community acquired pneumonia in adults: A conference report. Can J Infect Dis 1993;4:25-8.

2. Mandell LA, Marrie TI, Niederman MS, The Canadian Hospital Acquired Pneumonia Consensus Conference Group. Initial antimicrobial treatment of hospital acquired pneumonia in adults: a conference report. Can J Infect Dis 1993;4:317-21.

3. Marrie TJ. Community acquired pneumonia. Clin Infect Dis 1994;18:501-15.

4. Johanson WG, Pierce AK, Sanford JP, Thomas GD.
Nosocomial respiratory infections with Gram negative bacilli: the significance of colonization of the respiratory tract. Ann Intern Med 1972;77;701-6.

5. Fang GD, Fine M, Orloff J, et al. New and emerging etiologies for community-acquired pneumonia with implications for therapy. A prospective multicenter study of 359 cases. Medicine 1990;69:307-16.

6. Niederman MS, Bass JB, Campbell GD, et al. Guidelines for the initial management of adults with community acquired pneumonia: diagnosis, assessment of severity and initial antimicrobial therapy. Am Rev Respir Dis 1993;148:1418-26.

Lionel A Mandell MD FRCPC Professor of Medicine and Head, Division of Infectious Diseases, McMaster University, Hamilton, Ontario 


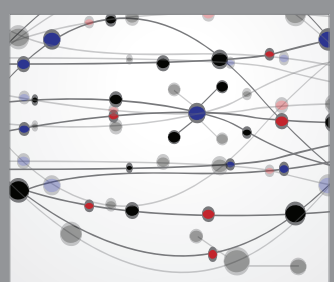

The Scientific World Journal
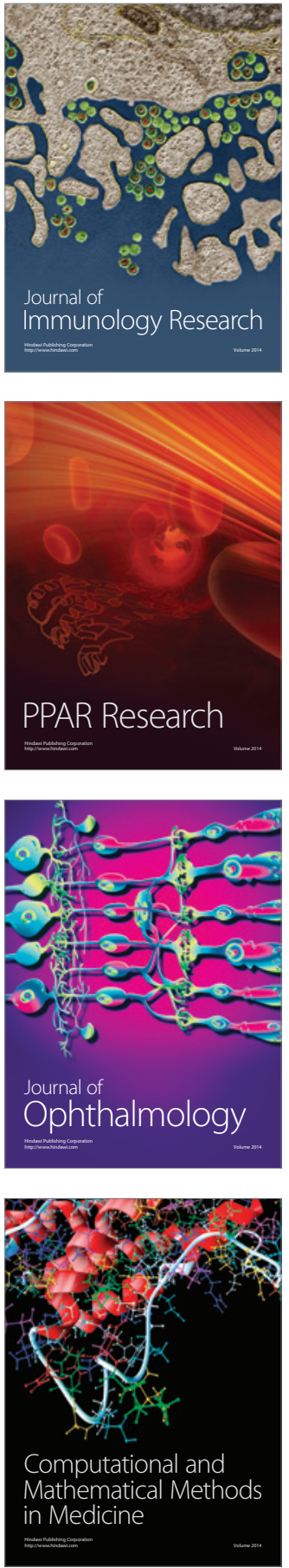

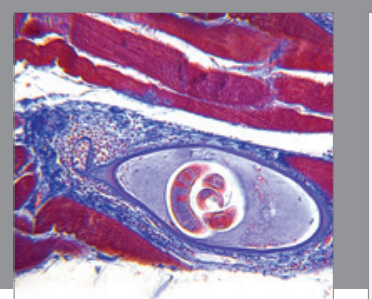

Gastroenterology Research and Practice

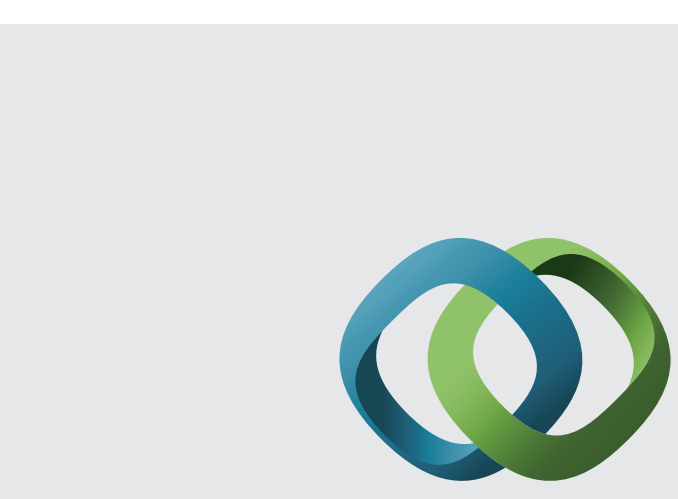

\section{Hindawi}

Submit your manuscripts at

http://www.hindawi.com
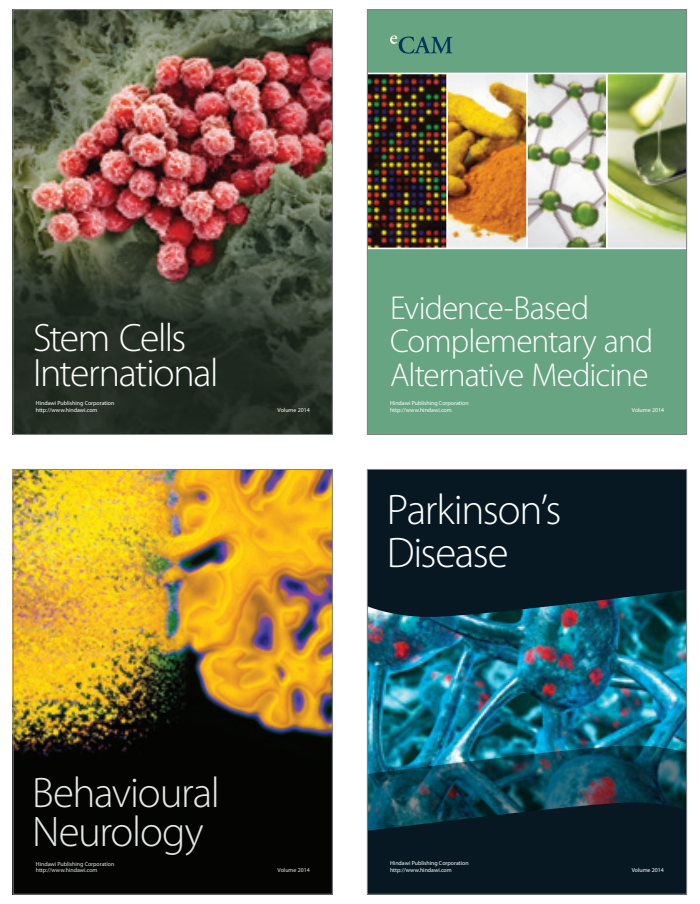
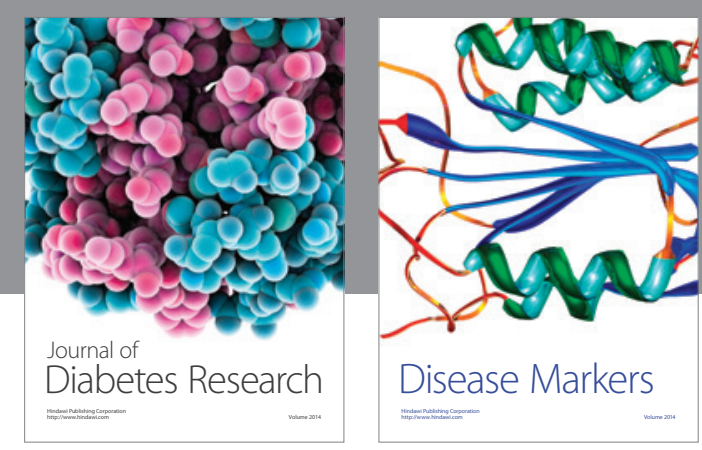

Disease Markers
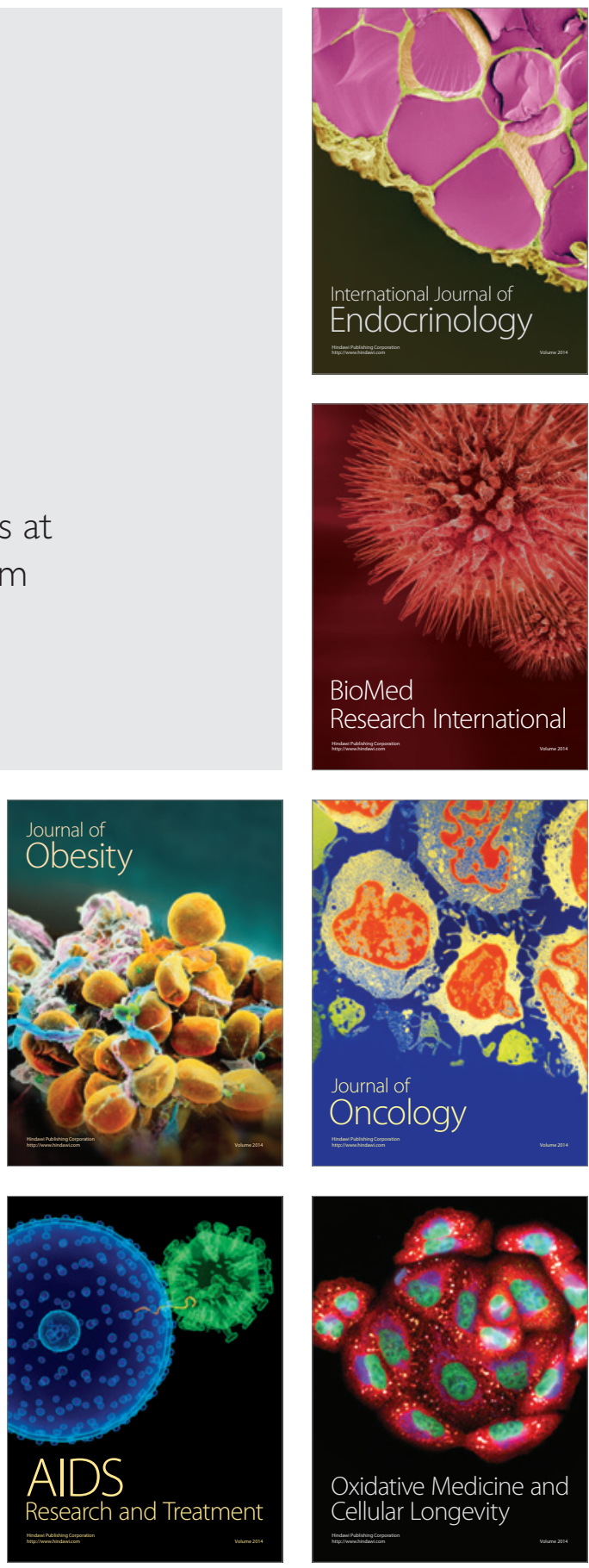